\title{
AN ENDOMORPHISM RING WHICH IS NOT ORE
}

\author{
WILLIAM D. BLAIR
}

\begin{abstract}
An example is given of a finitely generated module over a commutative ring whose endomorphism ring does not have a classical ring of quotients.
\end{abstract}

Vasconcelos [3] has shown that the endomorphism ring of a finitely generated module over a commutative noetherian ring has a classical ring of quotients. In [1] the author showed that the endomorphism ring of a finitely generated projective module over a commutative ring has a classical ring of quotients. The purpose of this note is to supply an example of a finitely generated module over a commutative ring whose endomorphism ring does not have a classical ring of quotients.

We recall that a ring $A$ has a (right) classical ring of quotients $Q$ if (i) $A \subseteq Q$, (ii) every regular element (nondivisor of zero) of $A$ is invertible in $Q$, and (iii) every element of $Q$ has the form $a b^{-1}$ where $a$ is an element of $A$ and $b$ is a regular element of $A$. A necessary and sufficient condition for a ring $A$ to have a right ring of quotients is for $A$ to satisfy the right Ore condition: Given $a, b$ in $A, b$ regular, then there exist $a^{\prime}, b^{\prime}$ in $A$, $b^{\prime}$ regular, such that $a b^{\prime}=b a^{\prime}$.

Let $R$ be a commutative ring and $I$ an ideal of $R$ such that:

(i) whenever $a \in R$ is regular then $a+i$ is regular for all $i \in I$;

(ii) $\operatorname{Ann}_{R}(I)=\{x \in R \mid x I=0\}=0$;

(iii) there exists $z \in R$ with $\operatorname{Ann}_{R}(z) \neq 0$ but $\operatorname{Ann}_{R / I}(\bar{z})=0$ where $\bar{z}=z+I \in R / I$. Let $M=R / I \oplus R . M$ is clearly a finitely generated $R$-module. We show that $A=\operatorname{End}_{R}(M)$ does not have a right classical quotient ring. Observe that

$$
A \approx\left[\begin{array}{ll}
\operatorname{End}_{R}(R / I) & \operatorname{Hom}_{R}(R, R / I) \\
\operatorname{Hom}_{R}(R / I, R) & \operatorname{End}_{R}(R)
\end{array}\right] .
$$

Let $f \in \operatorname{Hom}_{R}(R / I, R)$ then $0=f(\bar{l})=i f(\overline{\mathrm{I}})$ where $i \in I ; \operatorname{Ann}_{R}(I)=0$ implies $f(\overline{1})=0$ and hence $f=0$. Thus $\operatorname{Hom}_{R}(R / I, R)=0$. Also

$$
\operatorname{End}_{R}(R / I) \simeq \operatorname{End}_{R / I}(R / I) \simeq R / I \text { and } \operatorname{Hom}_{R}(R, R / I) \simeq R / I .
$$

Received by the editors September $11,1973$.

AMS (MOS) subject classifications (1970). Primary 16A08.

Key words and phrases. Endomorphism ring, classical ring of quotients.

(c) American Mathematical Society 1974 
Thus

$$
A \approx\left[\begin{array}{cc}
R / I & R / I \\
0 & R
\end{array}\right]
$$

If $\left[\begin{array}{ll}\bar{a} & b \\ 0 & c\end{array}\right]$ is regular in $A$ then $\bar{a}$ is regular in $R / I$ and $c$ is regular in $R$. Simple computation shows $\left[\begin{array}{ll}\bar{z} & 0 \\ 0 & 1\end{array}\right]$ is regular in $A$, where $z$ is the element of (iii) above.

We now show that $A$ does not satisfy the right Ore condition, for if it did then given $\left[\begin{array}{ll}\tilde{z} & 0 \\ 0 & 1\end{array}\right]$ and $\left[\begin{array}{ll}0 & \overline{1} \\ 0 & 0\end{array}\right]$ there would exist $\left[\begin{array}{cc}\bar{a} & b \\ 0 & c\end{array}\right]$ regular in $A$ and $\left[\begin{array}{ll}\bar{u} & \bar{v} \\ 0 & w\end{array}\right]$ in $A$ such that

$$
\left[\begin{array}{ll}
\bar{z} & 0 \\
0 & 1
\end{array}\right]\left[\begin{array}{ll}
\bar{u} & \bar{v} \\
0 & w
\end{array}\right]=\left[\begin{array}{ll}
0 & \overline{1} \\
0 & 0
\end{array}\right]\left[\begin{array}{ll}
\bar{a} & \bar{b} \\
0 & c
\end{array}\right],
$$

which says, in particular, that $\bar{z} \bar{v}=\bar{c}$, hence $z v=c+i$ for some $i \in I$. By property (i) $c+i$ is regular since $c$ is regular, thus $z v$ is regular and so $z$ is regular, a contradiction to the fact that $\operatorname{Ann}_{R}(z) \neq 0$.

It remains to show that there exists a ring $R$ with properties (i), (ii), and (iii). For this purpose let $F$ be a field and $S=F[X, Y] /\left(X^{2}, X Y\right)$ where $X$ and $Y$ are (commuting) indeterminants. Let $N=(X) /\left(X^{2}, X Y\right)$, $y=Y+\left(X^{2}, X Y\right) \in S$, and $\bar{y}$ the image of $y$ in $S / N$. We remark that $S / N \simeq$ $F[Y]$ and $\bar{y}$ corresponds to $Y$ under the isomorphism. $\operatorname{Ann}_{S}(y) \neq 0$ since $\left[Y+\left(X^{2}, X Y\right)\right]\left[X+\left(X^{2}, X Y\right)\right]=\left(X^{2}, X Y\right)$. However $\operatorname{Ann}_{S / N}(\bar{y})=0$, since $\operatorname{Ann}_{F[Y]}(Y)=0$. Since $N$ is a nil ideal $a+n$ is regular for any regular element $a \in S$ and any $n \in N$. Since $N$ is nilpotent we are still missing property (ii). We remedy this as follows: Let $x_{1}, x_{2}, x_{3}, \cdots$ be a countable set of (commuting) indeterminants and set

$$
R=S\left[x_{1}, x_{2}, \cdots\right] /\left(x_{1}^{2}, x_{2}^{3}, x_{3}^{4}, \cdots\right),
$$

where $S$ is the ring constructed above. Let

$$
I=\left(N \cdot S\left[x_{1}, x_{2}, \cdots\right]+\left(x_{1}, x_{2}, \cdots\right)\right) /\left(x_{1}^{2}, x_{2}^{3}, \cdots\right) .
$$

Clearly every element of $I$ is nilpotent and so property (i) holds. $\operatorname{Ann}_{R}(I)=$ 0 since if $a \in R$ such that $a I=0$ then $a$ must have $x_{1}, x_{2}^{2}, x_{3}^{3}, \cdots$ all as factors, an impossibility. Finally let $z$ be the image in $R$ of $y \in S$. Since $\operatorname{Ann}_{S}(y) \neq 0$, say $s y=0$ for $0 \neq s \in S$, then $\bar{s}=s+\left(x_{1}^{2}, x_{2}^{3}, \cdots\right) \neq 0$ in $R$ and $\bar{s} z=0$, so $\operatorname{Ann}_{R}(z) \neq 0$. Let $\bar{z}=z+I \in R / I$; we show $\operatorname{Ann}_{R / I}(\bar{z})=0$. Suppose $\bar{g} \in R / I$ and $0=\bar{z} \bar{g}$ where $g=s+h+\left(x_{1}^{2}, x_{2}^{3}, \cdots\right) \in R$ with $s \in S$ and $h \in\left(x_{1}\right.$, $\left.x_{2}, \cdots\right)$. Now $\bar{z} \bar{g}=0$ implies that $z g \in I$ that is $s y+y h \in N \cdot S\left[x_{1}, \cdots\right]+$ $\left(x_{1}, \cdots\right)$, which says $s y \in N$ since $s, y \in S$. But $\operatorname{Ann}_{S / N}(\bar{y})=0$ so $s \in N$ and hence $g \in I$, so $\bar{g}=0$ and $\operatorname{Ann}_{R / I}(\bar{z})=0$. 
As a final remark we observe that by Nagata's "principle of idealization" [2] we can form a new ring $R^{*}$ such that $M$ is an ideal of $R^{*}$ and also $\operatorname{End}_{R}(M) \simeq \operatorname{End}_{R^{*}}(M)$. This fact and the last example show that one cannot hope to generalize "finitely generated projective" in [1] to "finitely generated torsionless."

ACKNOWLEDGement. The author would like to thank Professor Lance W. Small for some helpful discussions which led to this example.

\section{REFERENCES}

1. W. D. Blair, Quotient rings of algebras which are module finite and projective, Acta Sci. Math. (Szeged).

2. M. Nagata, Local rings, Interscience Tracts in Pure and Appl. Math., no. 13, Interscience, New York, 1962. MR 27 \#5790.

3. W. V. Vasconcelos, Regular endomorphisms of finitely generated modules, J. London Math. Soc. (2) 4 (1971), 27-32. MR 45 \#246.

Department of Mathematical Sciences, Northern Illinois University, DeKalb ILLINOIS 60115 